\title{
Mundos del trabajo y relaciones de género: aportes y desafíos de una agenda de estudios interdisciplinar
}

\author{
Work worlds and gender relations: contributions and challenges of an \\ interdisciplinary study agenda
}

\section{(iD) Débora Garazi \\ deboragarazi@gmail.com \\ CONICET - Universidad Nacional de Quilmes - \\ Universidad Nacional de Mar del Plata, Argentina}

\section{Rosario Gómez Molla}

rosariogomezmolla@gmail.com

CONICET - Centro Interdisciplinario de

Investigaciones en Género, Instituto de Investigaciones

en Humanidades y Ciencias Sociales, Universidad

Nacional de La Plata, Argentina

Recepción: 09 Diciembre 2020

Aprobación: 12 Diciembre 2020

Publicación: 01 Marzo 2021

Cita sugerida: Garazi, D. y Gómez Molla, R. (2021). Mundos del trabajo y relaciones de género: aportes y desafíos de una agenda de estudios interdisciplinar. Descentrada, 5(1), e129. https://doi.org/10.24215/25457284e129

\begin{abstract}
Resumen: La introducción al dossier aborda algunos problemas académico-políticos clásicos dentro del campo interdisciplinario que estudia los mundos del trabajo y las relaciones de género. En el primer apartado, reconstruimos brevemente cómo se gestó este campo de estudios. En el segundo, identificamos tres nudos problemáticos de la agenda feminista: las discusiones en torno a la organización social del trabajo, dentro de la cual tiene un papel protagónico el trabajo doméstico y de cuidados; los procesos de generización y jerarquización de las profesiones; y los distintos modos en los que el Estado ha intervenido en torno a cuestiones relativas a trabajo y género. Por último, referimos a los "vacíos" que, entendemos, aún existen en este campo de estudios.
\end{abstract}

Palabras clave: Trabajo, Género, Profesiones, Trabajo doméstico, Trabajo remunerado y no remunerado.

\begin{abstract}
The introduction to the dossier addresses some classic academic-political problems within the interdisciplinary field that studies the world of work and gender relations. In the first section, we briefly reconstruct how this field of studies was created. In the second, we identify three problematic nodes of the feminist agenda: discussions on the social organization of work, within which domestic and care work plays a leading role; the processes of gendered and hierarchical professions and the different ways in which the State has intervened on issues related to work and gender. Finally, we refer to the "gaps" that, we understand, still exist in this field of studies.
\end{abstract}

Keywords: Work, Gender, Professions, Domestic work, Paid and unpaid work.

\section{INTRODUCCIÓN}

Según datos del Instituto Nacional de Estadísticas y Censos (INDEC), en el año 2020, en el marco de una crisis económica y social profundizada por la pandemia, la tasa de desocupación interanual que más se incrementó fue la de mujeres de 14 a 29 años, para las que creció del 23,4\% al 28,5\% (INDEC, 2020). Estos datos, sin embargo, no son una novedad. Históricamente, las mujeres -junto al colectivo LGBTQI+-fueron las más vulnerables dentro de los mundos del trabajo en diversos sentidos. No solo se concentraron en determinados sectores del mercado laboral con peores condiciones laborales y menores remuneraciones, sino 
que, además, sobre ellas recayó la responsabilidad de la realización del trabajo doméstico y de cuidados (Borderías y Carrasco, 1994; Barrère-Maurisson, 1991; Queirolo, 2020). Ahora bien, en la situación actual, ¿es preciso sostener que las mujeres están "desocupadas"? En un contexto en el que gran parte de las instituciones que garantizaban ciertos cuidados -las escuelas y las guarderías, por ejemplo- están cerradas y en el que las políticas de aislamiento y distanciamiento social resienten las tradicionales cadenas de cuidados, ¿podemos seguir sosteniendo que "aumentó la desocupación de las mujeres"? En todo caso, lo que sí aumentó, y de ello no hay dudas, es el desempleo y la extensión de formas de trabajo remunerado cada vez más informales y precarizadas. Sin embargo, las ocupaciones de las mujeres se multiplicaron, aunque los datos oficiales brindados por el Estado no den cuenta de ello ya que, a comienzos del siglo XXI, las instituciones encargadas de medir y registrar cuestiones relativas al trabajo continúan produciendo y reproduciendo nociones androcéntricas en las que prima su carácter remunerado y realizado en la esfera pública.

Frente a ello, los estudios de género, así como el movimiento feminista, demandan cada vez con mayor ímpetu una nueva y más equitativa organización social del trabajo. En ese sentido, este dossier propone abordar las relaciones y desigualdades de género en los mundos del trabajo desde una perspectiva interdisciplinaria que pone el foco, tanto en experiencias históricas como actuales y en trabajos remunerados, como no remunerados. Los artículos que lo componen invitan a reflexionar sobre cuestiones más amplias que trascienden los casos analizados y que los estudios de género han problematizado desde hace casi cinco décadas: la división sexual del trabajo, los estereotipos de género en el mercado laboral, la construcción de trabajos feminizados y masculinizados, los procesos de segregación laboral y discriminación salarial, las tensiones de género en los procesos de profesionalización, el trabajo reproductivo remunerado y no remunerado, y los trabajos de cuidados, entre otros.

Esta introducción no pretende reconstruir de forma exhaustiva los diálogos que se han dado entre los estudios de género y los estudios sobre el trabajo, dado que ya existen importantes balances que han dado cuenta de ellos (Andújar, 2017; Barrancos, 2005; Lobato, 2008; Palermo, 2008; Queirolo, 2004). Más bien, nos interesa abordar algunos problemas académico-políticos que han sido -y son- clásicos dentro de dicho campo interdisciplinario que, actualmente, forman parte de la agenda pública y que, además, enlazan a los artículos que conforman el presente dossier. En el primer apartado, reconstruimos brevemente cómo se gestó un campo de estudios interdisciplinario que aborda los mundos del trabajo desde una perspectiva de género y qué fue lo que dichos estudios evidenciaron. En el segundo, identificamos tres nudos problemáticos que forman parte de la actual agenda de investigación y de los colectivos que demandan mayores derechos para las mujeres y las disidencias: las discusiones en torno a la organización social del trabajo, dentro de la cual tiene un papel protagónico el trabajo doméstico y de cuidados; los procesos de generización y jerarquización de las ocupaciones y profesiones; y los distintos modos en los que el Estado ha intervenido en torno a cuestiones relativas al trabajo y género. Por último, referimos a los "vacíos" que, entendemos, aún existen en este campo de estudios para señalar posibles líneas de investigación a futuro.

\section{Trabajo y GÉNERo: Un CAMPO DE ESTUdiOS INTERDISCIPLINARIO}

Si bien hoy en día es indiscutible la participación histórica que han tenido las mujeres en los mundos del trabajo, hasta la década del ' 70 del siglo pasado, el trabajo femenino fue un tema relativamente descuidado por los estudios y análisis más tradicionales dentro del campo de las Ciencias Sociales. La gran cantidad de investigaciones que se produjeron desde entonces y, con mayor impulso en las últimas décadas, dieron cuenta de que, desde la modernidad, las mujeres fueron protagonistas casi exclusivas en la realización del trabajo doméstico o reproductivo y que, además, participaron de modo activo en la obtención de ingresos económicos para sus hogares (Pahl, 1991).

Si durante mucho tiempo el ámbito académico ha privilegiado como objeto de estudio una noción determinada de trabajo y de trabajadores, asociada al modelo industrial, masculino y asalariado, entendido 
como dominante desde fines del siglo XIX (Gorz, 1995), los estudios sobre las mujeres y los estudios feministas y con perspectiva de género han revolucionado este campo de estudios (Dauphin y Gardney, 2005). El análisis de la participación de las mujeres en los mundos del trabajo obligó no sólo a abrir el foco y observar otros sectores laborales, sino que puso en el centro del análisis una gama de relaciones de poder usualmente ignorada, reformuló los presupuestos de los que se partía y los problemas e interrogantes de investigación. Contribuyó, especialmente, a revisar, discutir y redefinir la propia categoría de trabajo, para poder incluir dentro de ella ciertas actividades que, hasta el momento, no eran conceptualizadas como tales.

Así pues, el concepto de división sexual del trabajo introdujo una dimensión sexuada a las relaciones de clase (Scott, 2008a). Esta noción evidenció una lógica que se sustenta sobre dos principios: el de separación de las tareas en trabajos de varones y de mujeres, y el de jerarquización de los primeros por sobre los segundos. Sin embargo, aunque la división sexual del trabajo permanece vigente, los modos concretos en los que se establece varían de acuerdo a una multiplicidad de factores (Hirata y Kergoat, 2007). La mirada de los estudios de género sobre los mundos del trabajo interroga esas especificidades, revelando las formas en las que la división sexual del trabajo se actualiza.

$\mathrm{Si}$, tradicionalmente, los mundos del trabajo -y los estudios que los abordaron- estuvieron permeados por el discurso de la domesticidad o doctrina de las esferas separadas, creador de concepciones de feminidad (mujermaternidad-mundo privado) y masculinidad (varón-trabajo remunerado-mundo público), los estudios de género proporcionaron las herramientas necesarias para cuestionar la naturalización de las identidades femeninas y masculinas, favoreciendo la reconstrucción de los contextos sociohistóricos en que tales identidades se desarrollaron y la superación del enfoque que asigna un espacio determinado a cada uno de los géneros (Nari, 2004; Lobato, 2007; Laufer, 2000).

De esta manera, una prolífica producción académica proveniente de distintas disciplinas ha contribuido a visibilizar el papel de las mujeres dentro de los mundos del trabajo -en el que incluimos tanto la esfera pública como privada-, resaltando las desigualdades y jerarquías de género existentes (Durán Heras, 2012; Sarti, Bellavitis, y Martini, 2018). Al mismo tiempo, evidenciaron que la participación femenina en ellos presentaba algunas especificidades, lo que permitía discutir las características asignadas al trabajo, al pensarlo desde nociones androcéntricas.

\section{Algunos Nudos PRoblemáticos DE LA AGENDA FEMINISTA}

Uno de los problemas que, desde distintas perspectivas, atraviesan a los artículos del presente dossier es el de la organización social del trabajo doméstico y de cuidados. Este tema, que hoy forma parte de la agenda pública, ha sido una preocupación clásica dentro del feminismo, tanto académico como militante (Laufer, Marry, Maruani, 2005; Carrasco, Borderías y Torns, 2011). En ese sentido, el clásico debate sobre el trabajo doméstico que tuvo lugar a mediados de los años '70 sirvió para hacer evidente su importancia, no solo para el mantenimiento y reproducción de la fuerza de trabajo y de las familias, sino para el funcionamiento del sistema económico (Davis, 2005; Federici, 2013; Rodríguez y Cooper, 2005).

Hasta el momento, la definición de trabajo asociada a la idea de una actividad remunerada había contribuido a excluir de las investigaciones a determinados trabajos y, en consecuencia, a los sujetos que los realizaban. Los estudios feministas y con perspectiva de género fueron los que comenzaron a discutir las tradicionales categorías marxistas de trabajo "productivo" (definido como el que se compra y vende en el mercado) y "reproductivo" (definido como el trabajo doméstico y de cuidados socialmente necesario para la reproducción de la fuerza de trabajo) (Carrasquer, Torns y Tejero, 1998). El objetivo principal era evidenciar el aporte de las mujeres a la producción, en tanto la asimilación de trabajo a su condición asalariada había implicado la negación del carácter productivo de todas las actividades que se realizaban de manera gratuita (Campillo, 2000; Cutuli, 2012). 
La ampliación de las fronteras del término 'trabajo', propuesta por el feminismo, constituyó un cambio significativo en relación a las tareas que podían incluirse dentro de dicha categoría. En ese sentido, el primer paso fue evidenciar que las tareas domésticas también eran trabajo y que las sujetas a las que, desde la modernidad, se les había asignado la responsabilidad de organizarlo y realizarlo eran, por lo tanto, trabajadoras -a pesar de no recibir una remuneración a cambio-. De esta manera, en el abordaje de las experiencias del trabajo femenino en el ámbito doméstico se volvió ineludible (Jelin, 1984; Aguilar, 2014; Sarti, Bellavitis y Martini, 2018).

Sin embargo, de la mano del crecimiento del sector de los servicios, muchas de las tareas que formaban parte del trabajo reproductivo realizado por las familias pasaron a formar parte de la esfera pública, ya sea a través de trabajos remunerados como voluntarios. Este es el caso del servicio doméstico, las guarderías infantiles, los geriátricos, las lavanderías, los restaurantes y otros servicios provistos por organizaciones de la sociedad civil o comunitarias (Allemandi, 2017; Pérez, Garazi y Cutuli, 2018; Borgeaud-Garciandía, 2018; Zibecchi, 2013). Pese a su inclusión en la esfera pública y remunerada, estas tareas no dejan de ser trabajo reproductivo en tanto que contribuyen a la reproducción, tanto individual como social de la vida. Así, se ha sugerido la sustitución de los conceptos de trabajo productivo y reproductivo por trabajo remunerado y no remunerado (Benería, 2006; Campillo, 2000).

En los últimos años ha crecido la preocupación en torno al trabajo específico de cuidado que, si bien durante mucho tiempo fue incluido dentro de la categoría de trabajo doméstico, presenta ciertas particularidades (Guerrero, Ramacciotti y Zangaro, 2019; Esquivel, Faur y Jelin, 2012; Rodríguez Enríquez, 2012). En dicha categoría, tanto la dimensión material del cuidado como la dimensión emocional y moral adquieren centralidad (Himmelweit, 2005; Martín Palomo y Damamme, 2020). Los estudios que se han focalizado en él han destacado las dificultades que existen para definir al concepto y su naturaleza multidimensional e interdisciplinaria (Araujo Guimarães e Hirata, 2020; Borgeaud-Garciandía, 2009).

Como señalan algunos artículos del dossier, estos trabajos están atravesados por dos importantes tensiones: su creciente mercantilización (con las implicancias que conlleva que una tarea tradicionalmente asociada a la esfera doméstica se traslade a la pública y se constituya como un empleo) y las dificultades de conciliación entre el trabajo remunerado y el trabajo doméstico de cuidados (Araujo Guimarães e Hirata, 2020; Pautassi y Zibecchi, 2013). Tanto los trabajos de cuidados remunerados como aquellos que se realizan de manera gratuita, están atravesados por clivajes de desigualdad, en los que el género se articula con la clase, la raza, la nacionalidad, la edad, dando lugar a una desigual distribución social de los mismos, en que las mujeres de sectores populares y, muchas veces, migrantes adquieren protagonismo, a pesar de que permanecen invisibles.

Asimismo, en el último tiempo ha comenzado a utilizarse el concepto de sostenibilidad de la vida como un concepto globalizador que, como ha señalado Cristina Carrasco Bengoa (2016), permite tener una visión en torno a de qué forma y con qué trabajos se satisfacen las necesidades humanas. Sin embargo, a pesar de la amplitud del término -que incluye la producción para el mercado, el ámbito ecológico y el de cuidados-, los esfuerzos se concentran en señalar al doméstico como el espacio privilegiado en el cual se proporcionan un conjunto de cuidados imposibles de evaluar con los parámetros del mercado.

Independientemente de las conceptualizaciones que se han desarrollado, lo cierto es que trabajo doméstico, de cuidados o de sostenibilidad de la vida, no son categorías neutras, sino que se anclan en experiencias generizadas. A pesar de posibles cambios que podrían estar produciéndose, la división sexual del trabajo, la organización de los tiempos de trabajo, así como la posibilidad de conciliar la participación en el mercado con las responsabilidades domésticas y de cuidados, sigue siendo una preocupación eminentemente femenina (Carrasco, 2003; Murillo, 2006).

La desigual distribución social del trabajo doméstico y de cuidados ha incidido en los modos en que las mujeres y hombres participan en el mercado de trabajo. En ese sentido, otro de los problemas que se ha abordado ha sido la generización de las ocupaciones y profesiones. En América Latina, en la primera década del siglo XXI, las investigaciones señalaban una ausencia de estudios sobre profesiones, ya fuera por privilegiar 
la sociología del trabajo (Panaia, 2006) o por englobar a los mismos en enfoques de un mayor nivel de abstracción (González Leandri, 2006). Sin embargo, de acuerdo con Graciela Queirolo y María Soledad Zárate Campos, el panorama actual es distinto y "si bien varios son los caminos que permitieron la expansión del campo, fue la historia del trabajo femenino uno de los más importantes” (2020, p. 16).

El problema de definir el concepto de profesión está presente en las discusiones de su campo de estudio. El sociólogo Eliot Freidson entiende que las dificultades de los estudios sobre profesiones radican en la pretensión de abordarlas "como si se tratara de un concepto genérico más que de un concepto que va cambiando con la historia" (2001, pp. 31-32). De modo que, para el autor, es importante pensar en casos particulares, delimitar el tiempo, el espacio, la ocupación y la perspectiva con la que se estudia para, desde allí, observar la manera concreta en que se la denomina profesión. En este sentido, el cruce entre los estudios sobre profesiones y los de género ilumina el modo en que las profesiones producen significados genéricos, al mismo tiempo que, a la inversa, los ideales de masculinidad y feminidad influyen en la conformación de aquéllas, así como también en las trayectorias profesionales individuales (Pozzio, 2012; Gómez Molla, 2017). Es decir, que los estudios de género han evidenciado la manera en la que en los procesos de profesionalización "la identidad de género de la persona se combinaba con la identidad de género de la ocupación" (Martin, Queirolo y Ramacciotti, 2019, p. 16). En otras palabras, los discursos y las prácticas atribuidos socialmente a cada uno de los géneros, se conjugan con un correlato equivalente en los discursos y las prácticas de ciertas tareas.

De este modo, la feminización y masculinización de algunas profesiones significan oportunidades laborales desiguales para mujeres y hombres. Como señala Joan Scott (2008b) en su trabajo sobre las historiadoras estadounidenses de fines del siglo XIX, no alcanza con observar el acceso de mujeres a determinadas profesiones, sino que es necesario indagar también en lo que sucedía una vez que estaban insertas en los grupos profesionales. Así, vemos que la feminización opera como oportunidad y como justificación del lugar subalterno que las mujeres ocupan en el mercado laboral (Biernat y Queirolo, 2018). Como oportunidad, porque en las ocupaciones feminizadas se promueve el empleo de mujeres, y como justificación de la desigualdad, porque se desconoce su formación para estas tareas con el argumento de que las habilidades que requieren son naturales de la condición femenina (Kergoat, 1994; Queirolo, 2018). Esta cuestión es central porque la capacitación es un elemento de distinción y jerarquización profesional (Queirolo y Zárate Campos, 2020). Por otro lado, mientras unas profesiones se feminizaron, otras cuentan, aun hoy, con una relativamente baja presencia femenina; es el caso, por ejemplo, de las ciencias “duras" (Rietti y Maffia, 2005) y las ingenierías (Panaia, 2014).

Pero la profesionalización no solo promueve desigualdades inter-género, sino que también lo hace al interior de cada grupo. La conciliación de las responsabilidades domésticas y el ejercicio profesional se apoya en los clivajes de desigualdad que ya mencionamos (clase, raza, nacionalidad, edad). Así, la profesionalización de unas depende de que puedan externalizar "sus" responsabilidades domésticas, mediante el empleo de mujeres que están en una condición más precaria (Hirata y Kergoat, 2007). Esta situación se refuerza con el déficit de políticas públicas destinadas a promover la redistribución de las responsabilidades domésticas y de cuidado y a ampliar los servicios que el Estado brinda en esta materia (Aspiazu, 2014). Ello nos lleva al tercer problema que atraviesa los textos del dossier: la intervención del Estado en los mundos del trabajo.

La respuesta de los estudios feministas a la pregunta “¿qué es el Estado?” puede ir desde una mirada que lo identifica como interlocutor neutral a otra que lo concibe como instrumento del poder patriarcal. Como plantea Adriana Valobra (2015), esto atraviesa también a la militancia feminista que articula demandas al Estado con acciones que van más allá de él o no lo involucran de manera directa. Ante este tipo de interpelaciones, los Estados se muestran oscilantes y contradictorios: hace al menos una década que los estudios sociales han problematizado la manera en que tradicionalmente se comprende al Estado -como un actor homogéneo, coherente y eficaz- para observarlo en su especificidad histórica, en sus múltiples agencias y en los diversos intereses que se dirimen en su conducción (Soprano, 2007). En otras palabras, en lugar de 
abordar al Estado en su conjunto, se focaliza en intervenciones estatales concretas, en nuestro caso en aquellas vinculadas a los mundos del trabajo y las relaciones de género.

En materia de trabajo y género, la conformación de una agenda sindical feminista interpela a los feminismos, a las trabajadoras, a los espacios sindicales y al Estado (Estermann, 2020; Rodríguez, 2020). En Argentina, la sanción en 2002 de la ley de Cupo Sindical Femenino fue importante -aun con sus limitacionespara incorporar mujeres en las negociaciones colectivas de trabajo y, con ellas, cláusulas de género en los convenios laborales (Aspiazu, 2014a). Es que la regulación del trabajo es central para entender la manera diferencial en que el Estado interviene en las trayectorias laborales de varones y mujeres (Anzorena, 2008). Históricamente, este contribuyó, a través de su normativa, a expandir los principios de excepcionalidad y complementariedad que caracterizaban el trabajo femenino asalariado (Queirolo, 2020). En la actualidad, el panorama es distinto pero, aunque hubo transformaciones, la desigualdad de género en materia laboral se mantiene también por acción y omisión del poder público.

Por otro lado, desde el punto de vista de las burocracias, observamos al Estado en su rol de empleador de mujeres y, por tanto, actor clave de la feminización de algunas ocupaciones; como los casos paradigmáticos de la docencia y la enfermería presentes en este dossier. Como contracara, están las fuerzas de seguridad, instituciones históricamente reacias a la incorporación de mujeres que han sido, solo en los últimos años, alcanzadas por políticas con perspectiva de género (Calandrón, 2019). Por último, el Estado y su comprensión - situada, heterogénea y contradictoria - de las relaciones de género, está presente en el punto de partida de la mayoría de las investigaciones, ya que las fuentes estatales (sobre todo las estadísticas) son primordiales para las Ciencias Sociales. En este punto, los estudios de género sobre los mundos del trabajo se enfrentan a problemáticas específicas: cómo se contaba el trabajo de las mujeres en los censos, cómo se mide el trabajo doméstico -si es que se mide-, cuál es la definición de trabajo implícita en los instrumentos estadísticos, etc. En este sentido, aunque el Estado como tal no es el objeto de estudio de este dossier, el alcance de la acción u omisión estatal en relación con el trabajo y las relaciones de género es un elemento relevante.

\section{SOBRE ESTE DOSSIER}

La interpelación de la militancia feminista y de los estudios de género a los mundos del trabajo transformó la comprensión sobre los mismos. El concepto de trabajo se redefinió, se cuestionaron sus premisas básicas y se ampliaron las fronteras que contenían un número reducido de sujetos, tareas y espacios vinculados a la experiencia laboral. En esta introducción nos propusimos dar cuenta de algunos hitos de este proceso, aún vigente, caracterizado por diálogos interdisciplinares que no se circunscriben exclusivamente al ámbito académico. Por eso retomamos tres problemas político-académicos que atraviesan las investigaciones con perspectiva de género y, también, la praxis feminista.

Ahora bien, los textos que componen este dossier, incluida esta introducción, posan la mirada en las experiencias laborales de mujeres; quedan, por lo tanto, en la sombra las de los varones y las de la población LGBTQI+. Sin embargo, esta omisión no fue intencional, sino que, en términos relativos estas experiencias aún ocupan un lugar menor dentro del campo de estudios interdisciplinarios sobre género y trabajo. Si bien dentro de los estudios sobre el trabajo, las experiencias masculinas han tenido centralidad, menos son las investigaciones que las han analizado desde una perspectiva de género. En nuestro país, estos estudios han focalizado en ocupaciones masculinizadas como las de los marítimos, ferroviarios, petroleros, azucareros, mozos y cocineros, entre otros. Dichas investigaciones han contribuido a problematizar sus identidades en tanto trabajadores varones, las cualificaciones y jerarquías tanto al interior del sector en que se desempeñaban como de la clase trabajadora en general, las experiencias cotidianas de trabajo y de sindicalización, los conflictos, etc. Asimismo, han dado cuenta de las nociones de masculinidad que subyacían a y se construían en dichas experiencias (Andújar, 2015; Caruso, 2016; D’Uva, 2019; Garazi, 2020; Gutiérrez, 2016; Palermo, 2009). Sin embargo, a pesar del importante crecimiento que en los últimos años han tenido estos estudios, el 
dossier presenta un vacío en ese sentido, lo que da cuenta de que aún predomina una noción de género que se entiende como sinónimo de mujeres.

En relación con el colectivo LGBTQI+, algunos estudios han señalado las discriminaciones que sufren las personas de dicho colectivo en el mercado laboral, que se manifiestan en la dificultad de acceso al empleo, en el hostigamiento, el acoso, las condiciones de trabajo desiguales y la inestabilidad laboral que caracteriza sus trayectorias (Berkins y Fernández, 2005; Berkins; 2007; Cutuli, 2015; Darouiche, 2017).

En ese sentido, posibles líneas de investigación podrían estar vinculadas con temas como las condiciones de trabajo de las personas del colectivo LGBTQI+, la vulneración, protección y promoción de sus derechos laborales, las prácticas discriminatorias, las estrategias de empoderamiento económico, las intervenciones institucionales en materia de derechos laborales de las personas LGBTQI+, su rol en la organización social del trabajo y en los trabajos domésticos y de cuidados, etc. A pesar de ciertos avances, aún está pendiente la conformación de una agenda de investigación en esa línea. Esta vacancia investigativa se presenta como deuda y, también, como promesa para seguir estudiando los modos en los que los mundos del trabajo, las relaciones de género y las identidades sexo-genéricas se conforman mutuamente.

Habiendo señalado el "vacío" que expone el dossier, pasamos a presentar los artículos que lo integran. Estos posan la mirada en distintos períodos, desde finales del siglo XIX a la actualidad, y en diversas latitudes de Brasil y Argentina. Abordan trabajos, sujetos y espacios diversos, y ofrecen, en esta pluralidad, la oportunidad de explorar desde distintos ángulos los nudos problemáticos que presentamos en estas líneas.

Los artículos de Laura Graciela Rodríguez, de Ana Paula Kondörfer y Karina Ramacciotti, y de Victoria Marquinez focalizan en la docencia, la enfermería y la ingeniería, respectivamente. Los textos interrogan distintas cuestiones vinculadas a las relaciones de género en cada una de estas profesiones. La formación, el prestigio social, la intervención del Estado y la dinámica interna del grupo profesional son algunos ejes que atraviesan los trabajos.

Laura Graciela Rodríguez focaliza en el período 1870-1914 para explorar la manera en que los discursos de género operaron en la ejecución y la legislación de la política pública vinculada a la docencia. El texto analiza tres variables: los programas de estudio de magisterio, los salarios de maestros y maestras, y la feminización de la docencia. Al historizar el devenir de estos elementos desde una perspectiva de género, la autora muestra que la docencia en el nivel primario no tuvo desde el comienzo una presencia casi exclusivamente femenina, que los salarios docentes no siempre fueron relativamente bajos y que los programas de estudio de magisterio variaron a lo largo del período.

El texto de Ana Paula Kondörfer y Karina Ramacciotti analiza el rol de la Fundación Rockefeller, institución filantrópica estadounidense, en la formación de enfermeras de Argentina y Brasil en la primera mitad del siglo XX. Las autoras estudian las estrategias de la fundación para difundir su mirada sobre la salud pública y las respuestas -bien diferentes entre sí- que ensayaron los gobiernos de ambos países. Así, el trabajo problematiza la profesionalización de la enfermería y el rol que desempeñó la Fundación Rockefeller en esos procesos, en el marco de determinadas relaciones internacionales entre Argentina y Brasil y Estados Unidos. El artículo muestra, también, a partir del estudio de algunas trayectorias individuales, el modo en que dicha profesionalización podía trascender el rol asistencialista de las enfermeras para darles un lugar en la planificación de la política de salud.

Si con la docencia y la enfermería nos referimos a profesiones que atravesaron procesos de feminización, el caso de la ingeniería es diferente, como observamos en el artículo de Victoria Marquinez. A partir de entrevistas a mujeres y varones que ejercen la ingeniería en la actualidad, en la ciudad de Mar del Plata, la autora explora el modo en que las identidades de género se combinan con las representaciones de género de la ingeniería en las trayectorias que analiza. Así, constata desigualdades en detrimento de las mujeres, provenientes del ordenamiento interno de los lugares de trabajo, como también de los arreglos domésticos de sus entrevistadas. Observa, además, algunas estrategias que, basadas en concepciones tradicionales o 
estereotipadas de las identidades femeninas, les permiten a las ingenieras posicionarse en sus trayectorias profesionales.

Los artículos de Marta Maria Valeriano, Tania Ludmila Dias Tosta y Jordão Horta Nunes, y de Victoria Mazzeo y Fabiana Bocchicchio problematizan cuestiones relativas a la organización social del trabajo y del cuidado. Ponen el foco en las relaciones y articulaciones entre el trabajo realizado para el mercado y el realizado para la familia a partir del análisis de diferentes experiencias que, además, permiten a las autoras problematizar cuestiones específicas.

El artículo de Valeriano, Dias Tosta y Horta Nunes focaliza en las experiencias de las trabajadoras domésticas remuneradas de Goiânia (Brasil) en la actualidad. A partir del análisis de datos de tipo cuantitativo y cualitativo, las autoras problematizan los arreglos domésticos que tienen lugar en los hogares de las trabajadoras y las identidades -ambiguas- que construyen en torno a su ocupación, sobre todo aquellas que viven en el hogar de sus empleadores. En su análisis, la condición de migrantes internas de la mayoría de las trabajadoras adquiere centralidad y se revela como un clivaje decisivo a la hora de abordar sus experiencias. De esta manera, el artículo muestra cómo, para este grupo particular de trabajadoras, las desigualdades de género se intersectan con las de clase, raza y origen migratorio, y se constituyen mutuamente.

El artículo de Victoria Mazzeo y Fabiana Bocchicchio examina la brecha de género en el uso del tiempo y, particularmente, en el trabajo reproductivo no remunerado de las mujeres de la Ciudad de Buenos Aires. A partir del análisis de encuestas de uso del tiempo, las autoras muestran que la desigual distribución de responsabilidades de cuidado dentro del hogar tiene consecuencias que trascienden la esfera doméstica y que gran parte de las desigualdades que afectan a las mujeres en el mercado de trabajo tienen allí su origen. El artículo, además, explora las desigualdades al interior del colectivo de mujeres y compara su situación según edad, nivel educativo, ingresos y composición del hogar.

De esta manera concluimos la introducción a este dossier con un agradecimiento a las autoras, cuyas contribuciones, oportunas y generosas, enriquecen el diálogo fructífero de los estudios interdisciplinarios sobre género y trabajo.

\section{REFERENCIAS}

Aguilar, P. (2014). El hogar como problema y como solución. Una mirada genealógica de la domesticidad a través de las politicas sociales. Argentina, 1890-1940. Buenos Aires: Ediciones del CCC.

Allemandi, C. (2017). Sirvientes, criados y nodrizas. Una aproximación a las condiciones de vida y de trabajo en la ciudad de Buenos Aires a partir del servicio doméstico (fines del siglo XIX yprincipios del XX). Buenos Aires: TeseoUniversidad de San Andrés.

Andújar, A. (2015). Comunidad obrera, género y políticas asistenciales: Comodoro Rivadavia, 1922-1932. Archivos del movimiento obrero y la izquierda, (7), 59-78. https://doi.org/10.46688/ahmoi.n7.135

Andújar, A. (2017). Historia social del trabajo y género en la Argentina del siglo XX: balance y perspectivas. Revista Electrónica de Fuentes y Archivos, 8(8), 43-59. Recuperado de https://refa.org.ar/file.php?tipo=Contenido\&id $=172$

Anzorena, C. (2008). Estado y división sexual del trabajo: las relaciones de género en las nuevas condiciones del mercado laboral. Utopia y Praxis Latinoamericana, 13(41), 47-68. Recuperado de http://ve.scielo.org/scielo.p hp?script=sci_arttext\&pid=S1315-52162008000200003\&lng=es\&tlng=es

Araujo Guimarães, N. y Hirata, H. (Eds.). (2020). El cuidado en América Latina: Mirando los casos de Argentina, Brasil, Chile, Colombia y Uruguay. Buenos Aires: Fundación Medifé Edita.

Aspiazu, E. (2014). Conciliación entre trabajo y responsabilidades familiares: una revisión teórica con enfoque de género. Investigium IRE: Ciencias Sociales y Humanas, V(1), 177-194. Recuperado de https://investigiumire.u nicesmag.edu.co/index.php/ire/article/view/88/66 
Aspiazu, E. (2014a). Equidad de género, mercado de trabajo y sindicalismo en la Argentina. Realidad Económica, (284), 10-36. Recuperado de http://nulan.mdp.edu.ar/2097/1/aspiazu.2014.pdf

Barrancos, D. (2005). Historia, historiografía y género: Notas para la memoria de sus vínculos en la Argentina. $L a$ aljaba, 9.

Barrère-Maurisson, M.-A. (1991). La división familiar de trabajo. La vida doble. Buenos Aires: Trabajo y Sociedad.

Benería, L. (2006). Trabajo productivo/reproductivo, pobreza y políticas de conciliación. Revista Nómadas, (24), 8-21. Recuperado de https://dialnet.unirioja.es/descarga/articulo/3995906.pdf

Berkins, L. y Fernández, J. (2005). La gesta del nombre propio: Informe sobre la situación de la comunidad travesti en la Argentina. Buenos Aires: Madres de Plaza de Mayo.

Berkins, L. (2007). Cumbia, copeteo y lágrimas; Informe nacional sobre la situación de las travestis, transexuales y transgéneros. Buenos Aires: A.L.I.T.T. Asociación de lucha por la identidad Travesti-Transexual.

Biernat, C. y Queirolo, G. (2018). Mujeres, profesiones y procesos de profesionalización en la Argentina y Brasil. Anuario del Instituto de Historia Argentina, 18(1), e060. https://doi.org/10.24215/2314257Xe060

Borderías, C. y Carrasco, C. (1994). Las mujeres y el trabajo: Aproximaciones históricas, sociológicas y económicas. En C. Borderías, C. Carrasco y C. Alemany, Las mujeres y el trabajo. Rupturas conceptuales. Madrid: Economía Crítica.

Borgeaud-Garciandía, N. (2009). Aproximaciones a las teorías del care. Debates pasados. Propuestas recientes entorno al care como trabajo. Revista Latinoamericana de Estudios del Trabajo (RELET), 14(22), 137-156. Recuperado de http://alast.info/relet/index.php/relet/article/view/196

Borgeaud-Garciandía, N. (Ed.). (2018). El trabajo de cuidado. Buenos Aires: Fundación Medifé Edita.

Calandrón, S. (2019). Minitas luchadoras: género y políticas públicas en estudios empíricos sobre las fuerzas policiales argentinas. Sudamérica: Revista de Ciencias Sociales, (11), 17-35. Recuperado de https://fh.mdp.edu.ar/revista s/index.php/sudamerica/article/view/3724

Campillo, F. (2000). El trabajo doméstico no remunerado en la economía. Revista Nómadas, (12), 98-115. Recuperado de https://www.redalyc.org/pdf/1051/105115263011.pdf

Carrasco Bengoa, M. C. (2016). Sostenibilidad de la vida y ceguera patriarcal. Una reflexión necesaria. Atlánticas, (1), 34-57.

Carrasco, C. (2003). ¿Conciliación? No, gracias. Hacia una nueva organización social. En M. I. Amoroso Miranda, A. Bosch Pareras, C. Carrasco Bengoa, H. Fernández Medrano y N. Moreno Sáenz, Malabaristas de la vida. Mujeres, tiempos y trabajos (pp. 16-34). Barcelona: Icaria.

Carrasco, C., Borderías, C. y Torns, T. (Eds.). (2011). El trabajo de cuidados: Antecedentes históricos y debates actuales. Madrid: Catarata.

Carrasquer, P., Torns, T., Tejero, E. y Romero, A. (1998). El trabajo reproductivo. Papers, (55), 95-114. Recuperado de https://www.raco.cat/index.php/Papers/article/viewFile/25507/25340

Caruso, L. (2016). Embarcados. Los trabajadores maritimos y la vida a bordo: Sindicato, empresas y Estado en el Puerto de Buenos Aires, 1889-1921. Buenos Aires: Imago Mundi.

Cutuli, R. (2012). Medir es conocer: Economía feminista y cuantificación del trabajo. Observatorio Laboral Revista Venezolana, 5(9), 23-41. Recuperado de http://nulan.mdp.edu.ar/1889/1/01488.pdf

Cutuli, M. S. (2015). Entre el escándalo y el trabajo digno. Etnografía de la trama social del activismo travesti en Buenos Aires (Tesis Doctoral). Universidad de Buenos Aires, Buenos Aires.

Davis, A. (2005). El trabajo doméstico toca su fin: Una perspectiva de clase. En Mujeres, raza y clase (pp. 221-239). Madrid: Akal.

D’Uva, F. (2019). Masculinidades obreras en los ferrocarriles: Fuerza física, riesgos y responsabilidad profesional en la Argentina de principios del siglo XX. Prohistoria. Historia, politicas de la historia, 9(25). Recuperado de https: //ojs.rosario-conicet.gov.ar/index.php/prohistoria/article/view/1009

Darouiche, C. A. (2017). Transformando la estructura de oportunidades: Una exploración sobre las desigualdades sociales, las oportunidades, la movilidad social del colectivo trans y la ilusión de un mejoramiento de las 
condiciones materiales de la vida. V Seminario Internacional Desigualdad y Movilidad Social en América Latina. Ensenada, Argentina. Recuperado de http://www.memoria.fahce.unlp.edu.ar/trab_eventos/ev.10683/ev.1068 3.pdf

Dauphin, C. y Gardney, D. (2005). Introducción. En J. Laufer, C. Marry, y M. Maruani, El trabajo del género. Las ciencias sociales ante el reto de las diferencias de sexo. Valencia: Germania.

Durán Heras, M. Á. (2012). Las fronteras entre el trabajo y el empleo. En El trabajo no remunerado en la economía global. Bilbao: Fundación BBVA.

Esquivel, V., Faur, E. y Jelin, E. (2012). Hacia la conceptualización del cuidado: Familia, mercado y estado. En Las lógicas del cuidado infantil. Entre las familias, el estado y el mercado (pp. 11-43). Buenos Aires: IDES.

Estermann, V. (2020). El sindicato por asalto. Feminismo y revitalización sindical en Argentina. En N. Goren y V. L. Prieto (Eds.), Feminismos y sindicatos en Iberoamérica (pp. 153-184). Ciudad Autónoma de Buenos Aires: CLACSO, UNPAZ. Recuperado de http://libraryl.nida.ac.th/termpaper6/sd/2554/19755.pdf

Federici, S. (2013). Revolución en punto cero: Trabajo doméstico, reproducción y luchas feministas. Madrid: Traficante de sueños.

Freidson, E. (2001). La teoría de las profesiones. Estado del arte. Perfiles educativos, 23(93), 28-43. Recuperado de h ttp://www.redalyc.org/articulo.oa?id=13209303

Garazi, D. (2020). El revés de las vacaciones: Hotelería, trabajo y género. Mar del Plata, segunda mitad del Siglo XX. Bernal: Editorial UNQ.

González Leandri, R. (2006). Campos e imaginarios profesionales en América Latina. Renovación y estudios de caso. Anuario IEHS, (21), 333-344. Recuperado de http://anuarioiehs.unicen.edu.ar/2006.html

Gómez Molla, R. (2017). Profesionalización femenina entre las esferas pública y privada. Descentrada, 1(1), e010. Recuperado de http://www.descentrada.fahce.unlp.edu.ar/article/view/DESe010

Gorz, A. (1995). Metamorfosis del trabajo. Búsqueda del sentido. Crítica de la razón económica. Madrid: Editorial Sistema.

Guerrero, G., Ramacciotti, K. y Zangaro, M. (2019). Los derroteros del cuidado. Bernal: Universidad Nacional de Quilmes.

Gutiérrez, F. (2016). Clase y masculinidad en la construcción de los derechos laborales y la división del trabajo azucarero. En A. Andújar, L. Caruso, F. Gutiérrez, S. Palermo, V. Pita, y C. Schettini, Vivir con lo justo. Estudios de historia social del trabajo en perspectiva de género. Argentina, siglo XIX y XX (pp. 131-154). Rosario: Prohistoria.

Himmelweit, S. (2005). El descubrimiento del trabajo no pagado. En D. Rodríguez y J. Cooper, El debate sobre el trabajo doméstico (pp. 251-277). México: UNAM.

Hirata, H. y Kergoat, D. (2007). Novas configurações da divisão sexual do trabalho. Cadernos de Pesquisa, 37(132), 595-609.

Instituto Nacional de Estadísticas y Censos. INDEC. (2020), Informes técnicos, 4(174). Recuperado de: https://www .indec.gob.ar/uploads/informesdeprensa/mercado_trabajo_eph_2trim20929E519161.pdf

Jelin, E. (1984). Familia y unidad doméstica: Mundo público y privado. Buenos Aires: CEDES.

Kergoat, D. (1994). Por una Sociología de las relaciones sociales. Del análisis crítico de las categorías dominantes a una nueva conceptualización. En C. Borderías, C. Carrasco y C. Alemany, Las mujeres y el trabajo. Rupturas conceptuales (pp. 517-531). Madrid: Economía Crítica.

Laufer, J. (2000). Introducción: Entre la esfera pública y la esfera privada, los retos de los derechos de las mujeres. En M. Maruani, C. Rogerat, y T. Torns (Eds.), Las nuevas fronteras de la desigualdad. Hombres y mujeres en el mercado de trabajo (pp.159-170). Barcelona: Icaria.

Laufer, J., Marry, C. y Maruani, M. (2005). El trabajo del género. Las ciencias sociales ante el reto de las diferencias de sexo. Valencia: Germania.

Lobato, M. (2007). Historia de las trabajadoras en la Argentina (1869-1960). Buenos Aires: Edhasa.

Lobato, M. (2008). Trabajo, cultura y poder: Dilemas historiográficos y estudios de género en Argentina. Estudios de Filosofia Práctica e Historia de las Ideas, 9(10), 29-45. 
Martín Palomo, M. T.y Damamme, A. (2020). Cuidados, en la encrucijada de la investigación. Cuadernos de Relaciones Laborales, 38(2), 205-216. Recuperado de https://revistas.ucm.es/index.php/CRLA/article/view/70878

Martin, A. L., Queirolo, G. y Ramacciotti, K. (2019). Mujeres y profesiones en la historia: un recorrido introductorio. En A. L. Martin, G. Queirolo, y K. Ramacciotti (Eds.), Mujeres, saberes y profesiones. Un recorrido desde las ciencias sociales (pp. 11-22). Ciudad Autónoma de Buenos Aires: Editorial Biblos.

Murillo, S. (2006). El mito de la vida privada: De la entrega al tiempo propio. Madrid: Siglo XXI.

Nari, M. (2004). Politicas de maternidad y maternalismo politico, 1890-1940. Buenos Aires: Biblos.

Pahl, R. E. (1991). Las divisiones del trabajo. Madrid: MTSS.

Palermo, S. (2008). El trabajo femenino en el siglo XX: nuevas miradas y planteos de la historia de la mujer y los estudios de género. Trabajos y Comunicaciones (2a Época), 8(34), 99-109. Recuperado de http://www.memoria.fahce.u nlp.edu.ar/art_revistas/pr.3725/pr.3725.pdf

Palermo, S. A. (2009). Masculinidade, conflitos e solidariedades no mundo do trabalho ferroviário na Argentina (1912-1917). Mundos do Trabalho, 1(2), 94-123. https://doi.org/10.5007/1984-9222.2009v1n2p94

Panaia, M. (2006). Una revisión de la Sociología de las Profesiones desde la teoría crítica del trabajo en la Argentina. Estudios del Trabajo, (32), 121-165. Recuperado de https://www.aset.org.ar/revista.php?rid=2

Panaia, M. (2014). La inclusión de la mujer en la profesión de ingeniería. Revista Virajes, 16(1), 19-43. Recuperado de https://revistasojs.ucaldas.edu.co/index.php/virajes/article/download/998/921

Pautassi, L. y Zibecchi, C. (2013). Las fronteras del cuidado: Agenda, derechos e infraestructura. Buenos Aires: Editorial Biblos.

Pérez, I., Cutuli, R. y Garazi, D. (2018). Senderos que se bifurcan. Servicio doméstico y derechos laborales en la Argentina del Siglo XX. Mar del Plata: Eudem.

Pozzio, M. (2012). Análisis de género y estudios sobre profesiones: propuestas y desafíos de un diálogo posible -y alentador-. Sudamérica: Revista de Ciencias Sociales, (1), 99-129. Recuperado de http://fh.mdp.edu.ar/ revistas /index.php/sudamerica/article/view/160/250

Queirolo, G. (2004). El trabajo femenino en la ciudad de Buenos Aires (1890-1940): Una revisión historiográfica. Temas de Mujeres, 1(1), 55-87. Recuperado de http://ojs.filo.unt.edu.ar/index.php/temasdemujeres/article/vi ew/4

Queirolo, G. (2018). Mujeres en las oficinas: trabajo, género y clase en el sector administrativo. Buenos Aires, 1910-1950. Ciudad Autónoma de Buenos Aires: Biblos.

Queirolo, G. (2020). Mujeres que trabajan. Labores femeninas, Estado y sindicatos (Buenos Aires, 1910-1960). Buenos Aires: Grupo Editor Universitario-Eudem.

Queirolo, G. y Zárate Campos, M. S. (2020). Mujeres, hombres y trabajo urbano. En G. Queirolo y M. S. Zárate Campos (Eds.), Camino al ejercicio profesional. Trabajo y género en Argentina y Chile (siglos XIX y XX) (pp. 11-26). Santiago de Chile: UAH Ediciones.

Rietti, S. y Maffía, D. (2005). Género, ciencia y ciudadanía. Arbor, 180(716), 539-544. https://doi.org/10.3989/ar bor.2005.i716.411

Rodríguez Enríquez, C. (2012). La cuestión del cuidado: ¿El eslabón perdido del análisis económico? Revista CEPAL, (106), 23-36.

Rodríguez, D. y Cooper, J. (2005). El debate sobre el trabajo doméstico. México: UNAM.

Rodríguez, T. (2020). Representación sindical y activismos feministas. El caso de la intersindical de mujeres. Revista Observatorio Latinoamericano y Caribeño, 4(I). Recuperado de https://publicaciones.sociales.uba.ar/index.php /observatoriolatinoamericano/article/view/5399/4858

Sarti, R., Bellavitis, A. y Martini, M. (Eds.). (2018). What is Work? Gender at the Crossroads of Home, Family, and Business from the Early Modern Era to the Present. Nueva York: Oxford.

Scott, J. W. (2008a). Sobre el lenguaje, el género y la historia de la clase obrera. En Género e Historia (pp. 77-94). México: Fondo de Cultura Económica. 
Scott, J. W. (2008b). Historiadoras profesionales en los Estados Unidos (1884-1894). En Género e Historia (pp. 221-244). México: Fondo de Cultura Económica.

Soprano, G. (2007). Del Estado en singular al Estado en plural: Contribución para una historia social de las agencias estatales en la Argentina. Cuestiones de Sociologia, (4), 19-48. Recuperado de http:// www.memoria.fahce.unlp .edu.ar/art_revistas/pr.3676/pr.3676.pdf

Valobra, A. M. (2015). El Estado y las mujeres, concepciones en clave feminista. Estudios Sociales del Estado, 1(2), 33 57. Recuperado de http://www.estudiossocialesdelestado.org/index.php/ese/article/view/44

Zibecchi, C. (2013). Organizaciones comunitarias y cuidado en la primera infancia: Un análisis en torno a las trayectorias, prácticas y saberes de las cuidadoras. Trabajo y Sociedad, (20), 427-447. 